University of Massachusetts Amherst

ScholarWorks@UMass Amherst

1990

\title{
Elimination of Double Peaks in the Iodimetric Flow Injection Visible Spectrophotometric Determination of Sulphite Using a Single-Channel Manifold
}

Julian Tyson

University of Massachusetts Amherst

A. G. Fogg

Loughborough University of Technology

X. Wang

Loughborough University of Technology

Follow this and additional works at: https://scholarworks.umass.edu/chem_faculty_pubs

Part of the Chemistry Commons

\section{Recommended Citation}

Tyson, Julian; Fogg, A. G.; and Wang, X., "Elimination of Double Peaks in the Iodimetric Flow Injection Visible Spectrophotometric Determination of Sulphite Using a Single-Channel Manifold" (1990). Analyst. 1306.

Retrieved from https://scholarworks.umass.edu/chem_faculty_pubs/1306 


\title{
Elimination of Double Peaks in the lodimetric Flow Injection Visible Spectrophotometric Determination of Sulphite Using a Single-channel Manifold
}

\author{
Arnold G. Fogg, Xiangwen Wang and Julian F. Tyson* \\ Chemistry Department, Loughborough University of Technology, Loughborough, Leicestershire \\ LE11 3TU, UK
}

\begin{abstract}
A flow injection method of determining sulphite with amperometric monitoring of iodine using a single-channel manifold in which iodine is formed in the reverse flow injection ( $\mathrm{rFI}$ ) manner and reacts with sulphite dispersing in the normal flow injection (nFI) manner has been adapted for use with visible spectrophotometry. The carrier stream consists of an alkaline solution containing iodate and an excess of iodide: injections of acid and then of acidified sulphite are made. The decrease in the iodine signal (measured at $352 \mathrm{~nm}$ ) in the presence of sulphite is proportional to the sulphite concentration in the injectate. The alkalinity of the carrier stream was adjusted to reduce the signal widths and to prevent the appearance of double peaks. A rectilinear decrease in signal size (down to ca. $10 \%$ of the signal size in the absence of sulphite) was obtained in the range $1 \times 10^{-5}-7 \times 10^{-4} \mathrm{M}$ sulphite using a single-channel manifold consisting of $3 \mathrm{~m}$ of $0.8 \mathrm{~mm}$ bore transmission tubing with a flow-rate of $5 \mathrm{ml} \mathrm{min-1}$ and an injection volume of $15 \mu \mathrm{l}$, when the carrier stream was $6.7 \times 10^{-6} \mathrm{M}$ in iodate, $6.7 \times 10^{-2} \mathrm{M}$ in iodide and $3.5 \times 10^{-3} \mathrm{M}$ in sodium hydroxide, and the sample solution was $0.1 \mathrm{M}$ in hydrochloric acid.
\end{abstract}

Keywords: Flow injection; sulphite determination; iodimetry; spectrophotometric detection; signal shape

Previously ${ }^{1}$ a flow injection (FI) amperometric method was recommended for the iodimetric determination of sulphite. Both the formation of iodine and its reaction with sulphite were carried out on-line in a single-channel manifold consisting typically of $3 \mathrm{~m}$ of $0.71 \mathrm{~mm}$ bore transmission tubing. Iodine was formed reproducibly in the reverse flow injection (rFI) manner in an injected bolus of dilute acid using a slightly alkaline potassium iodate eluent containing an excess of potassium iodide, and the iodine was monitored at a glassy carbon electrode held at $-0.20 \mathrm{~V}$ versus SCE, without interference from dissolved molecular oxygen. When acidified sulphite standard or sample solutions were injected into the same carrier stream a decreased iodine signal was obtained owing to reduction of iodine by the sulphite. The decrease in signal size was rectilinear with the sulphite concentration in the range $1 \times 10^{-5}-4 \times 10^{-4} \mathrm{M}$ when injections were made into a carrier stream $3 \times 10^{-5} \mathrm{M}$ in potassium iodate and $0.1 \mathrm{M}$ in potassium iodide. This paper describes the simple adaptation of this FI amperometric method to FI visible spectrophotometric determination but using information, gained since the former method was developed, on the shapes of FI signals. ${ }^{2,3}$

In the amperometric method the monitorand, iodine, is being formed in the rFI manner, and the signal shape is fundamentally different from that which is observed when the iodine is being formed in the normal flow injection (nFI) manner. ${ }^{1-3}$ That this should be so can be appreciated from the following considerations. When an iodate - iodide solution is injected into an acid carrier stream iodine will form only at the extremities of the injected bolus during the initial dispersion but the excess of acid soon reaches the centre of the bolus and all the injected iodate is converted into iodine and the conventional $\mathrm{nFI}$ signal is obtained. With further dispersion the peak decreases in size as the iodine is dispersed further. In the rFI situation (where acid is injected into an iodate - iodide carrier stream) an essentially infinite supply of iodate is present in the carrier stream, but the iodate (and hence the equivalent iodine) concentration at the centre of the bolus at the time of injection is zero. Iodine forms initially, as with nFI,

\footnotetext{
* Present address: Department of Chemistry, University of Massachusetts, Amherst, MA 01003, USA.
}

at the extremities of the bolus, but with increasing dispersion the equivalent concentration of iodate increases from the edges of the bolus inwards. A schematic diagram illustrating this concept of the formation of $\mathrm{nFI}$ and rFI signals is given elsewhere. ${ }^{4}$ Provided that the concentration of acid (the reagent in the bolus initially) does not become limiting during dispersion, the iodine concentration at the centre of the bolus increases to a limiting maximum value. Intuitively one can expect that rFI signals will be broad and that double peaks will be obtained more commonly during an rFI formation process than during an $\mathrm{nFI}$ formation process, and this was confirmed during recent studies on FI signal shapes. ${ }^{3}$ This situation is exacerbated when a monitorand (e.g., iodine) is formed in the rFI manner and then reacts with a determinand (e.g., sulphite) dispersing in the $\mathrm{nFI}$ manner, as the monitorand is removed primarily from the centre of the bolus whereas it is formed extensively over a wider region. This effect is apparent in our previous study in developing the amperometric method of determining sulphite (see Fig. 3 in reference 1). In that work the double peak, which was more apparent when sulphite was present, was made less obvious by increasing the flow-rate. In the present work the alkalinity of the iodate - iodide carrier stream was increased to decrease iodine formation at the extremities of the bolus, thus narrowing the iodine signal obtained. This was shown to have the effect of preventing the appearance of the double peak which otherwise appeared when sulphite was present in the injectate. Visible spectrophotometric studies are currently being carried out into the shapes of $\mathrm{nFI}$ and rFI signals. The preliminary findings have been discussed elsewhere. ${ }^{3}$

\section{Experimental}

A single-channel manifold incorporating a six-port rotary valve was used throughout. Slug injection $(15 \mu \mathrm{l})$ was used. The PTFE transmission tubing was of $0.8 \mathrm{~mm}$ bore and $3 \mathrm{~m}$ in

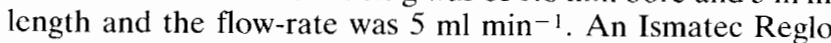
pump was used and the detector was a Pye Unicam SP-6-250 visible spectrophotometer fitted with a $10 \mathrm{~mm}$ path length $8-\mu \mathrm{l}$ quartz flow cell. Iodine was monitored at $352 \mathrm{~nm}$. 
In the rFI formation of iodine the reaction is never "complete" as iodate continues to be incorporated in the dispersing bolus from the carrier stream. Our work with the iodate - iodide - acid system has indicated that the signal increases rapidly with the iodide to iodate concentration ratio up to a ratio of about $10^{4}$, which was used here. Hence, in using this and similar methods all reagents should be weighed and reagent solutions made up to volume with reasonable precision. On the other hand re-calibration of FI methods is normally carricd out regularly, and this would normally be performed with new reagent solutions.

\section{Results}

Much of the optimisation of this procedure in terms of the production of the iodine signal and in determining sulphite had been carried out previously in connection with the amperometric method.' In the amperometric method the iodate - iodide carrier stream had been made slightly alkaline, as is normal analytical practice with this solution, to prevent

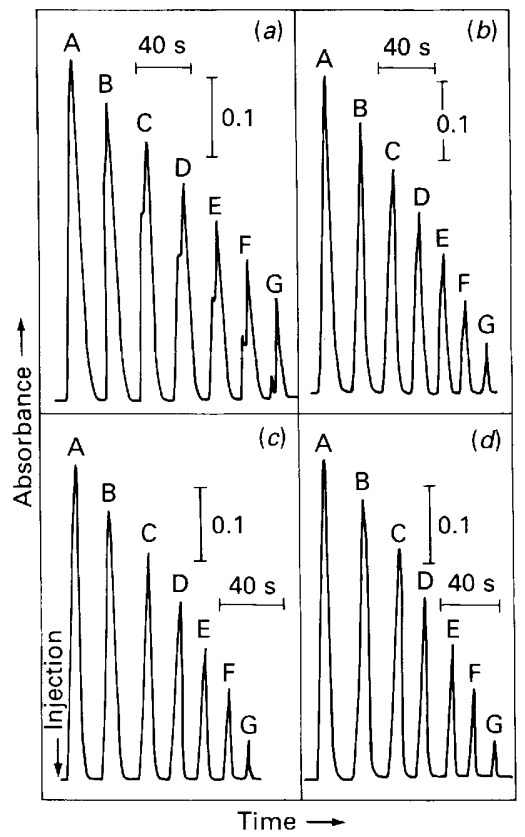

Fig. 1. Effect of increased alkalinity of the carrier stream on the signal shape and signal height obtained for the rFI formation of iodine and its reaction with sulphite dispersing in the $\mathrm{nFI}$ manner. Carrier stream: [iodate], $6.7 \times 10^{-6} \mathrm{M}$; [iodide], $6.7 \times 10^{-2} \mathrm{M}$; [sodium hydroxide], (a) $0.5 \times 10^{-3}$, (b) $2.5 \times 10^{-3}$, (c) $3.5 \times 10^{-3}$ and (d) $4.0 \times 10^{-3} \mathrm{M}$. Injectate: [hydrochloric acid], $0.1 \mathrm{~m}$; [sulphite]: A, 0 ; B, $1 \times 10^{-4} ; \mathrm{C}, 2 \times 10^{-4} ; \mathrm{D}, 3 \times 10^{-4} ; \mathrm{E}, 4 \times 10^{-4} ; \mathrm{F}, 5 \times 10^{-4} ;$ and $\mathrm{G}$, $6 \times 10^{-4} \mathrm{M}$

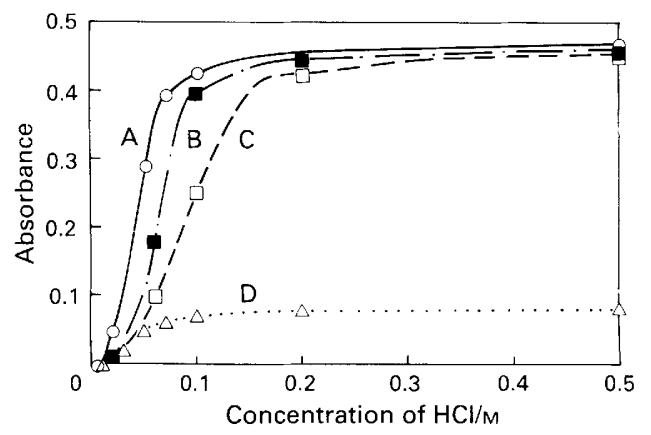

Fig. 2. Effect of the hydrochloric acid concentration of the injectate on the signals obtained. Carrier streams: A, B, C and D. A: [iodate], $6.7 \times 10^{-6}$; [iodide], $6.7 \times 10^{-2}$; and [sodium hydroxide], $3.0 \times$ $10^{-3}$ M. B: [iodate], $6.7 \times 10^{-6}$; [iodide], $6.7 \times 10^{-2}$; and [sodium hydroxide]. $3.5 \times 10^{-3} \mathrm{M}$. C: [iodate], $6.7 \times 10^{-6}$; [iodide], $6.7 \times 10^{-2}$; and [sodium hydroxide], $4.0 \times 10^{-3} \mathrm{M}$. D: [iodate], $1.3 \times 10^{-6}$; [iodide], $1.3 \times 10^{-2}$; and [sodium hydroxide], $2.0 \times 10^{-3} \mathrm{M}$ iodine forming in it due to residual acidity. In the study of signal shape, ${ }^{3}$ solutions that did not contain acid were adjusted to $\mathrm{pH} 11.0$ with sodium hydroxide. Clearly both the acidity of the injectate and the alkalinity of the carrier stream will affect the formation of iodine at the interface. In this work more attention was paid to studying the effect of the alkalinity of the carrier stream than was the case previously, and it was found, not surprisingly, that the signal width could be reduced by increasing the alkalinity of the carrier stream. This proved to be useful in reducing the incidence of double peaks. This can be seen in the sequence of signals shown in Fig. 1. The signals were obtained by injecting $15 \mu \mathrm{l}$ of $0.1 \mathrm{~m}$ hydrochloric acid or sulphite $\left(1 \times 10^{-4}-6 \times 10^{-4} \mathrm{M}\right)$ in $0.1 \mathrm{M}$ hydrochloric acid into carrier streams $6.7 \times 10^{-6} \mathrm{M}$ in iodate, $6.7 \times 10^{-2} \mathrm{M}$ in iodide and containing various concentrations of sodium hydroxide. For the injection of hydrochloric acid only, the double peak of the iodine signal is only just apparent at all the alkalinities studied: the signal is decreased slightly in size on increasing the alkalinity, but it can also be observed that the signal becomes narrower. In contrast, the effect of injecting acidified sulphite solutions is very marked at low alkalinities. With increasing concentration of sulphite the double-peaked nature of the signal becomes increasingly evident as the signal is split into a small front peak and the main rear peak. Increased

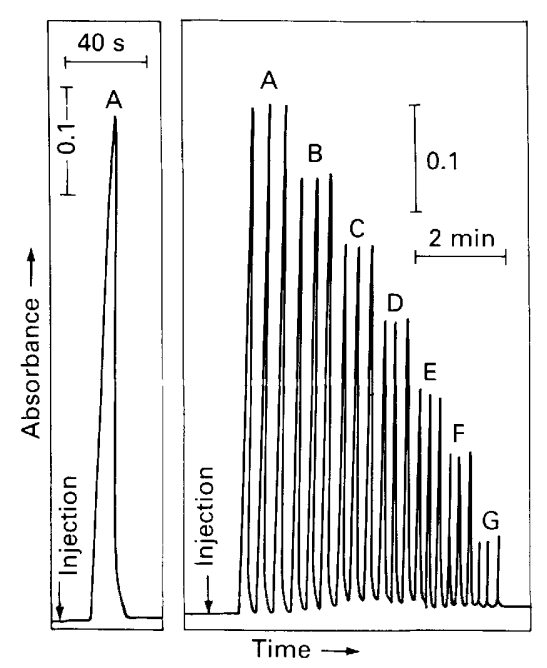

Fig. 3. Typical signals obtained for constructing a calibration graph under the optimised conditions. Transmission tubing, $3 \mathrm{~m}$ of $0.8 \mathrm{~mm}$ bore: and flow-rate, $5 \mathrm{ml} \mathrm{min}{ }^{-1}$. Carrier stream: [potassium iodate], $6.7 \times 10^{-6} \mathrm{M} ;$ [potassium iodide]. $6.7 \times 10^{-2} \mathrm{M}$; and [sodium hydroxide], $3.5 \times 10^{-3} \mathrm{M}$. Injectate: [hydrochloric acid], $0.1 \mathrm{~m}$; and [sulphite]: A, $0 ; \mathrm{B}, 1 \times 10^{-4} ; \mathrm{C}, 2 \times 10^{-4} ; \mathrm{D}, 3 \times 10^{-4} ; \mathrm{E}, 4 \times 10^{-4}$. $\mathrm{F}, 5 \times 10^{-4}$; and $\mathrm{G}, 6 \times 10^{-4} \mathrm{M}$

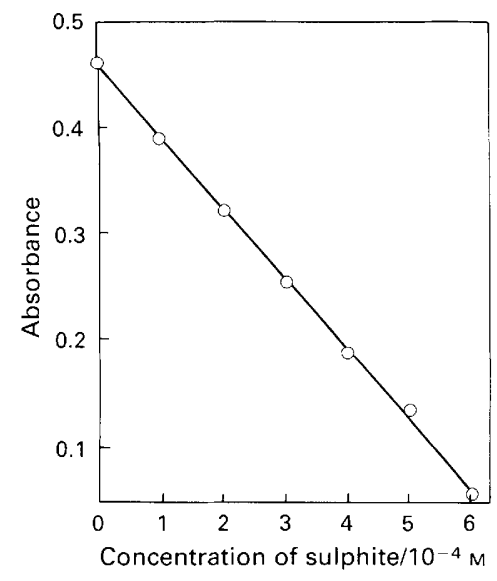

Fig. 4. Calibration graph obtained under the optimised conditions. Data were obtained from Fig. 3 
alkalinity has the effect of eliminating the front peak, but clearly the relative decrease in the size of the rear (main) pcak is greater at the higher alkalinities. Both of these effectsmust arise from the narrowing of the over-all signal.

Increasing the acid concentration of the injectate has the effect of increasing the signal width, and there is a greater chance of losing sulphur dioxide from the injectate solution before injection. The effect of the hydrochloric acid concentration of the injectate on the signal size at different carrier stream alkalinities is shown in Fig. 2. A combination of $0.1 \mathrm{~m}$ hydrochloric acid in the injectate and $3.5 \times 10^{-3} \mathrm{M}$ sodium hydroxide in the carrier stream was found to be a suitable compromise.

Typical signăls obtained for calibration purposes are shown in Fig. 3. A typical optimised system uses a carrier stream 6.7 $\times 10^{-6} \mathrm{M}$ in potassium iodate, $6.7 \times 10^{-2} \mathrm{M}$ in potassium iodide and $3.5 \times 10^{-3} \mathrm{M}$ in sodium hydroxide. The injectate is $0.1 \mathrm{~m}$ in hydrochloric acid. The following parameters were used: flow-rate, $5 \mathrm{ml} \mathrm{min-1;} \mathrm{injection} \mathrm{volume,} 15 \mu \mathrm{l}$; and transmission tubing, $3 \mathrm{~m}$ in length, $0.8 \mathrm{~mm}$ bore. The calibration graph obtained using these data is shown in Fig. 4. The coefficients of variation (six determinations) were found to be typically less than $2 \%$.

\section{Discussion}

The adaptation of the amperometric FI method for the determination of sulphite to visible spectrophotometric use was straightforward, but it was felt that the visible spectrophotometric method would be more acceptable and of more use to the average analytical chemist. Additionally, further experience and information were gained by working with $\mathrm{rFI}$ signals. Analytical chemists prefer assay signals to be single peaks, but because of the nature of the technique rFI is more prone to the formation of double peaks than is $\mathrm{nFI} .{ }^{3} \mathrm{We}$ have shown that with rFI, at sufficiently low injection volumes, dispersion from one boundary predominates and an apparently single peak is obtained. ${ }^{3}$ The position of this pcak is displaced from the position in which an nFI peak would appear and the fact that there is still a significant contribution from dispcrsion at the other boundary is clearly seen in the present system where sulphite dispersing in the nFI manner reacts with the iodine forming in the $\mathrm{rFI}$ manner. We have shown here how an rFI signal can be made "sharper" by adding what may be termed a "counter" reagent (hydroxide in this instance) to the carrier solution. It should be noted, however, that the point of maximum concentration of a substance being formed in the rFI manner is always displaced from that of a substance dispersing in the nFI manner. Because of this the use of hydroxide here to improve the appcarance of the signal is partly cosmetic in removing the small front peak. On the other hand its addition does remove some of the extraneous iodine signal from near the rear of the bolus: this is indicated by the greater effect of sulphite on the remaining iodine, i.e., the greater reduction in peak height.

As was found with the amperometric system it will be possible to increase the calibration range for the determination of sulphite by increasing the dispcrsion of the system, because with increasing dispersion the iodine control signal approaches a steady maximum value whereas the sulphite is increasingly dispersed and has a smaller effect on the iodine signal. Note that in these methods an excess of reagent (iodine) is monitored and that the methods are designed to detcrmine the determinand (sulphite) at much higher concentrations than the equivalent concentration of the reagent (iodate) present in the carrier stream which limits the amount of monitorand found. The spectrophotometric method described here determines approximately the same range of sulphite concentrations $\left(1 \times 10^{-5}-7 \times 10^{-4} \mathrm{M}\right)$ as does the previous amperometric method. ${ }^{1}$ Smaller concentrations of lodine (perhaps an order of magnitude less) could be determined both amperometrically and spectrophotometrically, which would allow slightly lower concentrations of sulphite to be determined but with lower precision.

Further studies are in progress on the shapes of FI signals. Preliminary work with the bromate - bromide - acid system has confirmed and extended the work with the iodine system. The bromine system has the advantage that bromide in acidic solution is not oxidised by dissolved oxygen. Hence it has been possible to show that for time-based large-volume $(2 \mathrm{ml})$ injections when an acidic bromate injectate is injected into an acidic bromidc carrier stream, and vice versa, the twin peaks of the signals are of equal size, which is not the situation when acid is only present in one solution. These studies will be published shortly ${ }^{4}$ and it is hoped that they will contribute significantly to the understanding of FI signals obtained using single-channel manifolds.

X. W. thanks the Government of the People's Republic of China and the Hubei University for financial support and leave of absence, respectively.

\section{References}

1. Fogg, A. G., Guta, C. W., and Chamsi, A. Y., Analyst, 1987. 112, 253

2. Fogg, A. G., Analyst, 1986, 111, 859.

3. Fogg, A. G., Wang, X., and Tyson, J. F., Analyst, 1989, 114, 1119

4. Fogg, A. G., Cipko, E., Farabella, L., and Tyson, J. F., Analyst, in the press 